\title{
Health Care Associated Cutaneous Abscess - A Rare Form of Primary Gonococcal Infection
}

\author{
Jasmina Vraneš ${ }^{1,2}$, Ivana Lukšić², Jasna Knežević ${ }^{2}$, Sunčanica Ljubin-Sternak ${ }^{1,2, *}$ \\ ${ }^{1}$ Medical Microbiology Department, School of Medicine, University of Zagreb, Zagreb, Croatia \\ ${ }^{2}$ Clinical Microbiology Department, Teaching Institute of Public Health “Dr Andrija Štampar”, Zagreb, Croatia \\ *Corresponding author: sljsternak@stampar.hr
}

Received January 31, 2015; Revised February 14, 2015; Accepted February 25, 2015

\begin{abstract}
A rare form of extragenital gonococcal infection was recorded after accidental exposure in laboratory during the identification of Neisseria gonorrhoeae. Three days after needlestick injury, on the left index finger of exposed technician very painful abscess developed. Beside local symptoms presented on the finger, fever or any other signs or symptoms of infection have not been observed. Oral antibiotic therapy with azithromycin was administered, and upon appearance of pus in the center of erythematous area surgical incision was performed. After incision the abscess was healed.
\end{abstract}

Keywords: gonococcus, cutaneous abscess, laboratory-acquired infection

Cite This Article: Jasmina Vraneš, Ivana Lukšić, Jasna Knežević, and Sunčanica Ljubin-Sternak, "Health Care Associated Cutaneous Abscess - A Rare Form of Primary Gonococcal Infection.” American Journal of Medical Case Reports, vol. 3, no. 3 (2015): 88-90. doi: 10.12691/ajmcr-3-3-10.

\section{Introduction}

Gonorrhea is well known sexually transmitted disease (STD) caused by gram negative diplococcus Neisseria gonorrhoeae ( $N$. gonorrhoeae). Gonococcus primarily affects the mucosa, most often genital, but extragenital mucous membranes (rectal, pharyngeal, and conjunctival) can be primarily infected as well. Cutaneous manifestation of infection may also occur, usually as a result of disseminated infection [1-3]. Primary, cutaneous, gonococcal infection is extremely rare, and it is always the result of the inoculation of bacteria through the damaged skin.

Health care-associated gonococcal infections have also been described [4]. These infections have presented as conjunctivitis as result of either direct finger-to-eye contact or exposure to splashes of either liquid cultures or contaminated solutions [5,6]. Here we report laboratoryacquired, gonococcal infection as a result of accidental needlestick injury.

\section{Case Presentation}

A 24-year-old male technician reported needlestick injury acquired three days ago while he worked on characterization of $N$. gonorrhoeae strain isolated from patient with urethritis. Injury happened on Friday, at the end of the working process in diagnostic laboratory. He was wearing the gloves, however, needle pierced the glove and stubbed to the skin of his left hand index finger. Immediately upon the injury he went to the sink and thoroughly washed the wound while vigorously squeezing the finger to express as much blood as he could. Additionally, the wound was disinfected with $70 \%$ ethanol. Despite those post exposure measures, during the weekend he developed very painful local abscess accompanied with erythema and induration. Tehnician was afebrile and no other signs or symptoms of infection was present. Otherwise, he was healthy person without any underlying condition, symptoms or history of STDs.

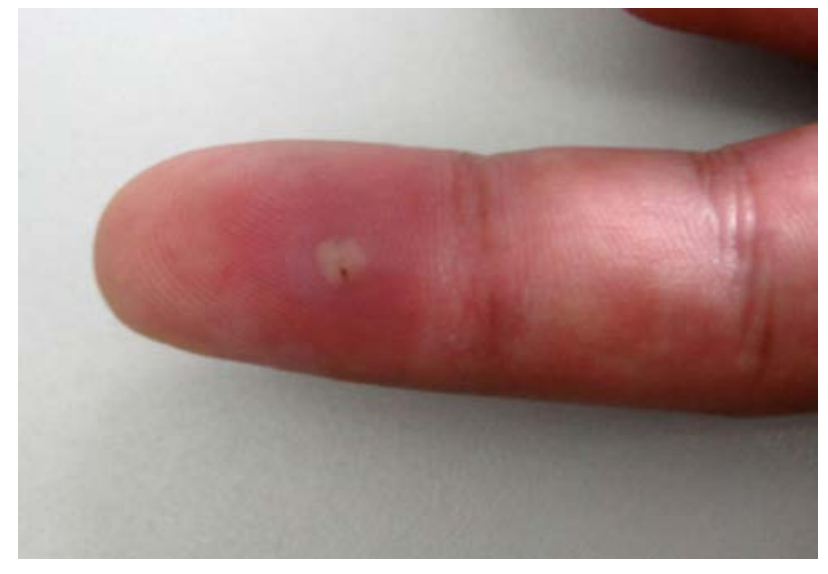

Figure 1. Cutaneous gonococcal abscess of index finger - purulent center surrounded by painful erythematous induration

Oral antibiotic therapy with azithromycin (500 mg daily over three days) was immediately started. The following day puss collection in the center of erythematous area was observed (Figure 1). Therefore, on the fourth day following the accident, incision of the abscess was indicated and performed.

During the surgical treatment of the abscess, two swabs were taken for microbiology analysis. One swab was used 
in preparation of Gram and Methylene blue stained microscopic slides, and typical intracellular diplococci were observed (Figure 2).

The other swab was used for molecular detection of $N$. gonorrhoeae. Molecular detection was chosen due to poor quality of specimen for cultivation after surgical incision, and higher sensitivity and specificity of molecular method compared to classical procedure.

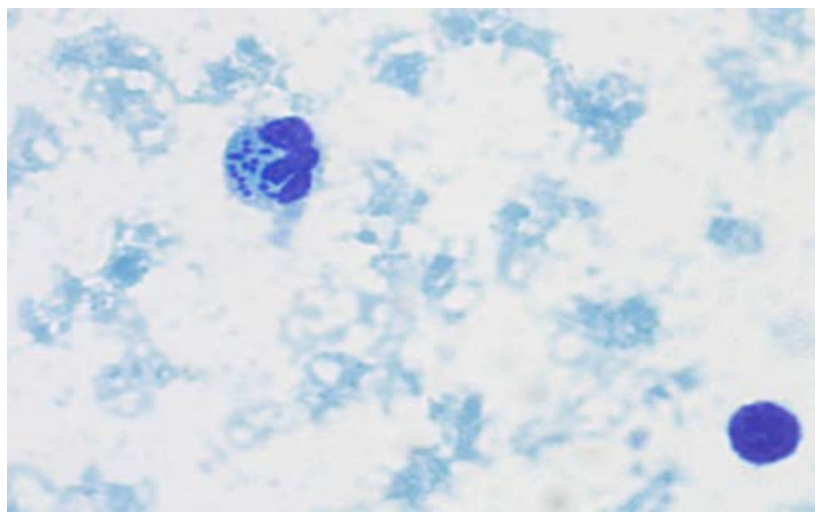

Figure 2. Methylene blue stain of pus specimens obtained from incisional drainage of the index finger showing typical diplococci within leukocytes

Real-time polymerase chain reaction was conducted using LightMix ${ }^{\circledR} \quad$ Kit Neisseria gonorrhoeae (TIB MOLBIOL GmbH, Berlin, Germany) with LightCycler ${ }^{\circledR}$ FastStart DNA Master HybProbe (Roche Diagnostics $\mathrm{GmbH}$, Manhiem, Germany) on the LightCycler ${ }^{\circledR} 480$ II Instrument (Roche Diagnostics GmbH, Manhiem, Germany). Molecular test revealed clearly positive result (Figure 3).

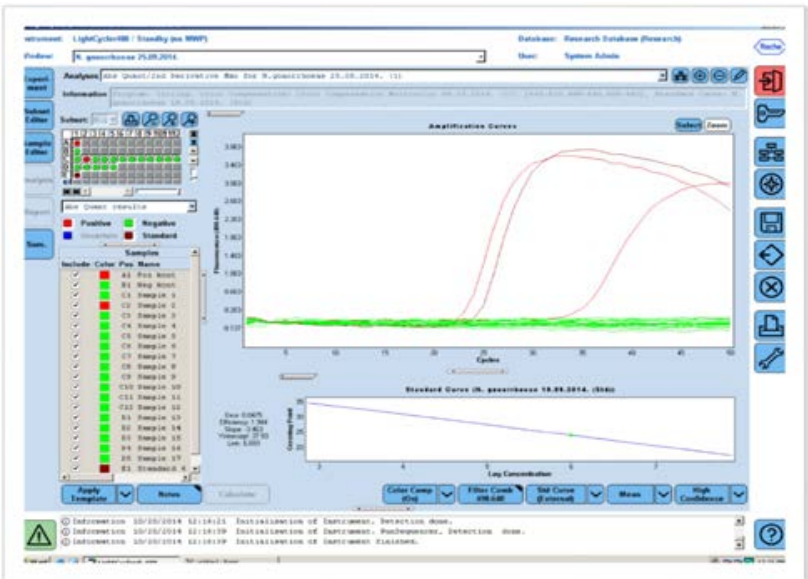

Figure 3. Real-time PCR result showing three amplification curves: positive control, standard, and positive pus specimen at position $\mathrm{C} 2$

After incision, wound was left widely open to enable effective drainage. One week after incision the abscess completely healed.

\section{Review of Literature \& Discussion}

Cutaneous abscess of genitourinary region is not an unexpected complication of genital gonococcal infection and it may occur with or without a coexisting genital infection. Gonococcal abscess of the prepuce, Tyson's glands and penile corpus cavernosum have been observed
$[7,8,9]$. Perianal gonococcal abscess without urethritis in homosexual male patient also has been described [10].

Extragenital location of solitary or multiple gonococcal abscesses is almost always result of hematogenic spread of $N$. gonorrhoeae from distant site of primary infection [11] Disseminated gonococcal infection commonly presents with subcutaneous abscess, arthritis and pyrexia [12], but any organ or tissue can be affected (i.e. unusual case of man with epidural gonococcal abscess) [13].

Primary cutaneous abscess caused by $N$. gonorrhoeae outside the genital area is extremely rare. Pathogenesis of these infections usually involves direct inoculation of pathogens or exposure of injured skin to contaminated secretions, since gonococci cannot adhere to intact skin. Several cases of gonococcal scalp abscess in infants after intrauterine monitoring during delivery have been reported [14], as well as the case of gonococcal abscess on the abdominal wall of 22-month-old boy that originated from contact with an infected adult [15].

Primary, gonococcal abscess localized on finger, has been described in adults. Presumably, man got the infection from an infected woman as a result of digitalgenital contact [16]. Previous trauma of men's finger and damaged skin barrier seems to be the requirement for achieving primary gonococcal infection at such atypical localization [17].

Laboratory-associated gonococcal infections were also described, but most of them were result of conjunctival exposure to contaminated material and therefore presented as conjunctivitis [4-6]. In present case direct inoculation of $N$. gonorrheae by needlestick injury manifested as cutaneous abscess of traumatized finger. Although, the particular strain of $N$. gonorrhoeae involved in this accident was $\beta$-lactamase negative, azithromycin was administrated regarding the recommendations for treatment of gonococcal infections [18], and the route of administration. Additionally, incision was performed the day after receiving the first dose of azithromycin. Surgical incision and drainage is the definitive treatment of a soft tissue abscess [19]. Antibiotics alone are ineffective in the face of a localized collection of pus. Combination of surgical incision and antibiotic therapy resulted in faster and complete recovery.

\section{Conclusion}

Impairment of skin barrier is the key factor in pathogenesis of primary extragenital gonococcal cutaneous abscess. Treatment of gonococcal abscess requires evacuation and drainage of the pus accompanied with appropriate antibiotic therapy.

\section{References}

[1] Jitmuang A, Boonyasiri A, Keurueangkul N, Leelaporn A, Leelarasamee A. Gonococcal subcutaneous abscess and pyomyositis: a case report. Case Rep Infect Dis. 2012; 2012: 790478.

[2] Ho YM1, Sebastin SJ, Lim AY. Culture-negative hand abscesses in immunocompetent individuals. Singapore Med J. 2012; 53(2):e38-9.

[3] Ghosn SH, Kibbi AG. Cutaneous gonococcal infections. Clin Dermatol. 2004; 22:476. 
[4] Wilson DE, and Chosewood CL Eds, Biosafety in Microbiological and Biomedical Laboratories. US Government Printing Office, Washington DC. Revised 5th Edition; 2009.

[5] Bruins SC, Tight RR. Laboratory acquired gonococcal conjunctivitis. JAMA. 1979; 241: 274.

[6] Zajdowicz TR, Kerbs SB, Berg SW, Harrison WO. Laboratoryacquired gonococcal conjunctivitis: successful treatment with single-dose ceftriaxone. Sex Transm Dis. 1984; 11(1): 28-9.

[7] Fiumara NJ, Eisen R. Cutaneous gonococcal abscess: a case report. Sex Transm Dis. 1984; 11(1): 34-5.

[8] Kar HK, Sharma PK, Arora TC, Sabhnani S. Gonococcal Tyson's gland abscess \& urethral stricture with genital wart \& herpes genitalis: a case report. Indian J Sex Transm Dis. 2005;26(1):36-7.

[9] Sagar J, Sagar B, Shah DK. Spontaneous penile (cavernosal) abscess: case report with discussion of aetiology, diagnosis, and management with review of literature. TheScientificWorldJOURNAL. 2005; 5: 39-41.

[10] El-Dhuwaib Y, Ammori BJ. Perianal abscess due to Neisseria gonorrhoeae: an unusual case in the post-antibiotic era. Eur J Clin Mirobiol Infect Dis. 2003; 22(7): 422-3.
[11] Gomperts BN, White LK. Gonoccocal hand abscess. Pediatr Infect Dis J. 2000; 19(7): 671-2.

[12] Oboth Owino N, Goldmeier D, Wall RA. Gonooccal septicaemia presenting as a subcutaneous abscess. Br J Vener Dis. 1981; 57 : 143-4.

[13] van Hal SJ, Post JJ. An unusual cause of an epidural abscess. Med J Aust. 2004; 180(1): 40-1.

[14] Asnis DS, Brennessel DJ. Gonococcal scalp abscess: a risk of intrauterine monitoring. Clin Pediatr (Phila). 1992; 31(5): 316-7.

[15] Dickson SD, Alter SJ, Cutaneous gonococcal abscess of the abdomen in a child. Pediatr Emerg Care. 2011; 27(9): 863-4.

[16] Fitzpatrick JE, Gramstad ND, Tyler H Jr. Primary extragenital cutaneous gonorrhea. Cutis. 1981; 27(5):479-80.

[17] Scott MJ Jr, Scott MJ Sr. Primary cutaneous Neisseria gonorrhoeae infections. Arch Dermatol. 1982; 118:351-2.

[18] CDC. Update to CDC's Sexually Transmitted Diseases Treatment 2010 Guidelines: Oral Cephalosporins No Longer a Recommended Treatment for Gonococcal Infections. MMWR 2012; 61(31): 590-4.

[19] Roberts JR, Hedges JR. Clinical Procedures in Emergency Medicine, 5th ed., Saunders/Elsevier, Philadelphia, 2010. 